\title{
Stochastic Collocation for Device-level Variability Analysis in Integrated Photonics
}

\author{
Yufei Xing, ${ }^{1}$ Domenico Spina, ${ }^{2}$ Ang $\mathrm{Li}^{1}{ }^{1}$ Tom Dhaene, ${ }^{2}$ and Wim Bogaerts ${ }^{1,3, *}$ \\ ${ }^{1}$ Photonics Research Group, Department of Information Technology, \\ Center for Nano and Biophotonics, Ghent University imec, Ghent B-9000, Belgium \\ ${ }^{2}$ Department of Information Technology, Internet Based Communication Networks and Services (IBCN), \\ Ghent University iMinds, Gaston Crommenlaan 8 Bus 201, B-9050 Gent, Belgium \\ ${ }^{3}$ Luceda Photonics, 9200 Dendermonde, Belgium
}

compiled: January 27, 2016

\begin{abstract}
We demonstrate the use of stochastic collocation to assess the performance of photonic devices under the effect of uncertainty. This approach combines high accuracy and efficiency in analyzing device variability with the ease of implementation of sampling-based methods. Its flexibility makes it suitable to be applied to a large range of photonic devices. We compare the stochastic collocation method to a Monte Carlo technique on a numerical analysis of the variability in silicon directional couplers.
\end{abstract}

OCIS codes: (130.3120) Integrated optics devices; (000.5490) Probability theory, stochastic processes, and statistics; (230.7370) Waveguides

http://dx.doi.org/10.1364/XX.99.099999

\section{Introduction}

Integrated Photonics, and in particular, silicon photonics, is rapidly enabling complex photonic functions on a chip [1]. However, variability due to fabrication processes and operational conditions limits the complexity of the circuits that can be implemented $[2,3]$. A proper analysis of the effects of the variability in geometrical, electrical and optical parameters on the performance of the photonic building blocks and circuits has become crucial. Indeed, the variability introduced by the variations of the manufacturing process is a primary source of degradation of larger photonic circuits, especially when wavelength-selective filters are implemented [4]. The primary functional parameters that are affected are the waveguide propagation constants (the effective index) and the coupling coefficients in coupling structures.

The Monte Carlo (MC) method [5] is considered the standard approach for variability analysis, thanks to its accuracy and ease of implementation. Unfortunately, the MC analysis has a slow convergence rate, and it requires a large number of data points (simulations or measurements). Therefore, MC has a very high computational cost, considering that accurate simulations of photonic devices can be time and resource intensive.

The generalized polynomial chaos (gPC) expansion has been applied in several domains as an efficient alternative to the classic MC method [6-8] and, recently,

\footnotetext{
* Corresponding author: wim.bogaerts@ugent.be
}

it has been proposed for the variability analysis of photonic devices $[9,10]$.

The gPC-based modeling approach aims at expressing a stochastic process as a series of orthogonal basis functions with suitable coefficients and gives an analytical representation of the variability of the system on the random variables under consideration [11].

In this paper, we propose a stochastic collocation (SC) method as an efficient alternative to characterize photonic devices under the effect of uncertainty. The fundamental principle of the SC approach is to approximate the unknown stochastic solution by interpolation functions in the stochastic space. The interpolation is constructed by repeatedly solving (sampling) the deterministic problem at a pre-determined set of nodes in the stochastic space. This approach offers similar high accuracy and efficiency as the stochastic gPC method, but at the same time, it is easy to implement, like samplingbased methods (e.g. MC approach).

We apply the SC method to analyze the variability of a key building block for Silicon photonic circuits: the directional coupler. This device is essential in the construction of wavelength filters, as it implements an arbitrary fractional $2 \times 2$ power coupling, but at the same time, it is extremely sensitive to fabrication variations: a small shift in linewidth or thickness of the core can dramatically change the coupling coefficients.

The paper is organized as follows: Section 2 gives a general introduction of SC methods. It provides the essential mathematics knowledge that readers need to 
know to understand its application in the photonic domain. Section 3 uses directional coupler as an example to test the performance of SC in performing variability analysis of photonic devices. Section 4 draws the conclusions.

\section{Stochastic Collocation Methods}

SC methods are based on interpolation schemes to compute stochastic quantities. The interpolation is constructed by repeatedly solving (sampling) the deterministic problem at a pre-determined set of nodes in the stochastic space [12] (also defined as collocation points). Indeed, a stochastic process $\mathbf{Y}(\boldsymbol{\xi})$ can be expressed as

$$
\mathbf{Y}(\boldsymbol{\xi})=\sum_{i=1}^{Q} \mathbf{Y}\left(\boldsymbol{\xi}_{i}\right) L_{i}(\boldsymbol{\xi})
$$

where $\boldsymbol{\xi}$ denote the $N$ stochastic parameters and $\left\{L_{i}(\boldsymbol{\xi})\right\}_{n=1}^{N}$ represents the interpolation basis functions.

For a photonic device, the process $\mathbf{Y}$ could correspond to the functional parameters such as the waveguide propagating constants and the coupling coefficients in coupling devices. The stochastic variables $\xi_{i}$ correspond to device properties affected in a stochastic way by fabrication and operational conditions (e.g. waveguide line, width or temperature).

In (1) different types of interpolation schemes can be adopted (e.g. piecewise linear [12, 13], Lagrange [11, 14] or interpolation methods that belong to the general class of positive interpolation operators, like multivariate simplicial methods [15]). However, the key issue for this approach is the selection of the support nodes, such that using the minimal number of nodes one achieves a good approximation.

For example, if the Lagrange interpolation scheme is chosen, the element $L_{i}$ in (1) for a one-dimensional interpolation can be expressed as

$$
L_{i}(\xi)=\prod_{i=1, i \neq j}^{Q} \frac{\xi-\xi_{i}}{\xi_{j}-\xi_{i}}
$$

where $L_{i}$ is equal to 1 for $\xi=\xi_{j}$ and is equal to 0 for $\xi=\xi_{i}$. Next, for interpolation in multiple dimensions, a tensor-product approach can be used and equation (1) becomes

$\mathbf{Y}(\boldsymbol{\xi})=\sum_{i_{1}=1}^{Q_{k_{1}}} \cdots \sum_{i_{N}=1}^{Q_{k_{N}}} \mathbf{Y}\left(\xi_{i_{1}}^{k_{1}}, \ldots, \xi_{i_{N}}^{k_{N}}\right)\left(L_{i_{1}}^{k_{1}} \otimes \cdots \otimes L_{i_{N}}^{k_{N}}\right)$

where $\xi_{i}^{k}$ is the $i$-th node in the $k$-th direction and the total number of nodes used in (3) is

$$
Q=\prod_{n=1}^{N} Q_{k_{n}}
$$

As it can be seen from (4), the number of nodes required by the full tensor product increases rapidly with the number of random parameters $N$. For example, if three random variables are considered and 10 collocation points are used for each parameter, a total of 1000 nodes are required by the full tensor product approach. Hence, the performance of the photonic device under study must be evaluated for 1000 different combinations of the random variables considered, leading to an expensive computational time.

The required number of nodes can be significantly reduced by adopting sparse grids in the stochastic space, based on the Smolyak algorithm [12, 16-19]. By choosing the collocation points correctly, Smolyak algorithm drastically reduces the total number of nodes used in the interpolation with respect to the full tensor product approach while preserving a high level of accuracy.

It is important to remark that the SC models are expanded using interpolation functions of independent random variables $\boldsymbol{\xi}$ [11]. In the general case of correlated random variables, decorrelation can be obtained via a variable transformation, such as the Nataf transformation [20] or the Karhunen-Loéve expansion [21].

The stochastic moments (mean, variance, ...) can be computed utilizing analytical formulas and then very efficiently, once the analytical form of the interpolation functions $\left\{L_{i}(\boldsymbol{\xi})\right\}_{n=1}^{N}$ has been decided. For example, if the random variables $\boldsymbol{\xi}$ are defined in the sample space $\boldsymbol{\Omega}$, the mean of $\mathbf{Y}(\boldsymbol{\xi})$ is defined as

$$
\mu(\mathbf{Y}(\boldsymbol{\xi}))=\int_{\Omega} \mathbf{Y}(\boldsymbol{\xi}) W(\boldsymbol{\xi}) d \boldsymbol{\xi}
$$

where $W(\boldsymbol{\xi})$ is the joint probability density function (PDF) of the random variables $\boldsymbol{\xi}$. Using equation (1) in (5) leads to

$$
\mu(\mathbf{Y}(\boldsymbol{\xi}))=\int_{\boldsymbol{\Omega}} \sum_{i=1}^{Q} \mathbf{Y}\left(\boldsymbol{\xi}_{i}\right) L_{i}(\boldsymbol{\xi}) W(\boldsymbol{\xi}) d \boldsymbol{\xi}
$$

which depends only on the interpolation functions $L_{i}(\boldsymbol{\xi})$ and joint PDF $W(\boldsymbol{\xi})$. Note that, if the choice of the interpolation functions and probability measure does not allow an analytical computation of the stochastic moments like (6), an efficient numerical solution can be used (e.g. by MC analysis of the interpolation model or numerical integration). Finally, it is important to remark that it is not possible to define a priori the speed-up of a generic SC modeling technique compared to the $\mathrm{MC}$ method. Indeed, the number of nodes needed to compute an accurate SC model (which is directly related to the efficiency of SC methods, as described above) cannot be decided upfront, since depends on the following factors:

- the impact of the chosen random variables $\boldsymbol{\xi}$ on the variations of the stochastic process considered $\boldsymbol{Y}$ (dynamic stochastic processes require a higher number of collocation points); 
- the interpolation scheme $L_{i}$ adopted (the more powerful the interpolation scheme, the fewer nodes are needed);

- the sampling strategy adopted (efficient sampling strategy limit the number of collocation points used);

- the number of random variables considered (the higher the number of variables the more collocation points are needed).

However, it has been proven in the literature that, for a limited number of random variables (indicatively less than ten) SC methods are much more efficient with respect to the MC analysis, see [11, 12, 14]. For stochastic processes depending on a high number of random variables, the efficiency of SC methods is significantly reduced.

Two approaches can be used to increase the efficiency of an SC modeling technique. Using nested sampling schemes allows to adaptively choose the collocation points (additional details are provided in Section 3.E and Appendix 5.B). Adopting adaptive sparse grids [16] reduces the nodes requirement, which is especially useful when a high number of random variables is considered. For a more detailed reference on SC methods, we refer the reader to $[11,12,14,16]$.

\section{Directional Coupler Example \\ 3.A. Benchmark Description}

We demonstrate the use of SC for integrated photonics through the analysis of a directional coupler in a silicon photonics platform. Silicon photonics is rapidly gaining adoption because its potential for large-scale integration and volume manufacturing. [22]. However, the same high index contrast that enables dense integration also makes silicon photonic waveguides extremely sensitive to small imperfections in their geometry. Also, the high thermo-optic coefficient of silicon makes silicon photonic devices very temperature sensitive. For instance, a change in linewidth and thickness of waveguide would vary effective index $\left(n_{e f f}\right)$ of optical modes noticeably, resulting in a shift of $1 \mathrm{~nm}$ in the response of a wavelength-selective filter. This variation leads to performance degradation in devices such as directional couplers, Mach-Zehnder interferometers (MZI) and ring resonators, and limits the number of devices that a single circuit can have.

Power coupling devices are essential parts for splitting and combining power in photonic circuits. Among them, directional couplers (DC) are widely used for their simplicity in layout and easy-to-understand operation. An advantage of directional couplers compared to other $2 \times 2$ couplers is that the coupling ratio can be continuously adjusted by choosing the length of the coupling section. Furthermore, DCs constitute the building blocks of many larger photonic devices such as rings, MZIs and so on.
A DC consists of two parallel waveguides and connecting bend sections: the light in a single waveguide is mostly confined in the silicon core, but an exponentially decaying field extends into the cladding. When two waveguides are brought in proximity, the modes of the two waveguides couple and form two supermodes with opposite symmetry (an even and an odd mode). The beating of these supermodes translates in a sinusoidal power transfer from one waveguide to the next and back along the propagation axis $z$. The power coupling $K(z)$ in a DC can be expressed as [23]

$$
K(z)=\sin ^{2}\left(\kappa z+\kappa_{0}\right)
$$

The power coupling consists of two parts: the contribution of the straight waveguide $\kappa z$ and of the bend section $\kappa_{0}$. Bend part will introduce an initial phase in the coupling term. Usually, the bend contribution is fairly small, and the power coupling is sinusoidally varying with the waveguide coupling section length $z$.

The rate of coupling is defined as the field coupling coefficient $\kappa$, which is determined by the geometry of the coupler cross-section, such as the waveguide width, thickness, and gap between the waveguide cores.

Let us assume that, for simplicity, the two waveguides in a DC are identical. As a result, the straight section of the DC layout is defined by three parameters: the waveguide width $w$, thickness $t$ and gap $g$ (Fig. 1). Furthermore, we assume that, in the lithography process, the centers of the two waveguides are located at the designed position. It is a good assumption for optical lithography techniques, but might be less accurate for e-beam written devices. With this assumption, the sum of the gap $g$ and $2 \times$ the half-waveguide width $w$ is constant, as shown in Fig. 1. Therefore, in our example, we can describe the full geometry of the directional coupler with only two parameters: $w$ and $t$.

In this study, we will use the SC technique to find out how geometry variability influences the DC performance, namely the coupling coefficient $k$. Indeed, due to the fabrication variations, the fabricated linewidth $w$, thickness $t$ and gap $g$ are different on the value chosen during the design phase. To prove the robustness and modeling power of the proposed approach, we assume the width $w$ and thickness $t$ of the DC as correlated random variables, rather than independent, following the Gaussian distribution. It is not an unrealistic assumption: thickness variations could induce over-etching on the sidewalls.

It is good to note that the SC methods can deal with random variables with arbitrary distribution. It is therefore not necessary that the $t$ and $w$ adhere to a Gaussian distribution.

\section{B. Simulation Setup}

According to the theory of supermodes, we can write the coupling coefficient $\kappa$ as [23] 

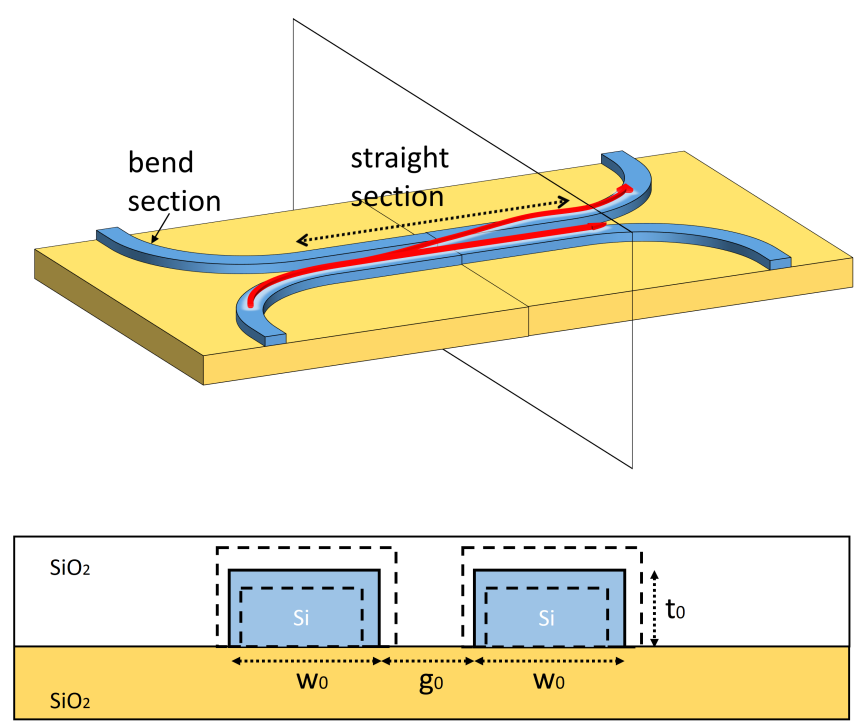

Fig. 1: The upper plot shows the perspective view of a symmetric DC. Red arrows present the flow of light. Part of the light is coupled from bottom waveguide to the above one. The cross section is amplified in the lower plot. The mean width and thickness of the DC are $w_{0}$ and $t_{0}$, respectively. The width $w$ and thickness $t$ of the fabricated DC are indicated as dashed boxes.

The refractive indexes are $n_{s i}=3.44, n_{\mathrm{SiO}_{2}}=1.45$.

$$
\kappa=\frac{\pi}{\lambda}\left(n_{\text {eff-o }}-n_{\text {eff-e }}\right)
$$

where $n_{e f f_{-o}}$ and $n_{e f f_{-} e}$ are the effective index of asymmetrical and symmetrical supermodes in DC. For our silicon photonics devices, we assume the wavelength to $\lambda=1.55 \mu \mathrm{m}$. Next, the nominal value of the width and thickness are $w_{0}=450 \mathrm{~nm}$ and $t_{0}=220 \mathrm{~nm}$, respectively, while we fix the sum of width $w$ and gap $g$ at 650 nm.

To calculate $\kappa$ of a given geometry, we define the DC structure accordingly and simulate $n_{e f f_{-} o}$ and $n_{e f f_{-} e}$ in the mode solver Fimmwave using its Film Matching Mode (FMM) solver. For later performance comparisons, all simulations are performed on a computer with an Intel Core i5 2500 quad-core CPU clocked at $3.3 \mathrm{GHz}$ and $8 \mathrm{~GB}$ of memory.

\section{C. Problem Definition}

As mentioned in 3.A, we considered the coupling coefficient $K$ of a directional coupler as a stochastic process depending on two correlated random variables with Gaussian PDFs: the width $w$ and the thickness $t$. Hence, the joint PDF of the two random variables considered is defined as

$$
W_{\boldsymbol{\eta}}=\frac{1}{2 \pi \operatorname{det}(\mathbf{C})^{\frac{1}{2}}} \exp \left(-\frac{1}{2}(\boldsymbol{\eta}-\boldsymbol{\mu})^{T} \mathbf{C}^{-1}(\boldsymbol{\eta}-\boldsymbol{\mu})\right)
$$

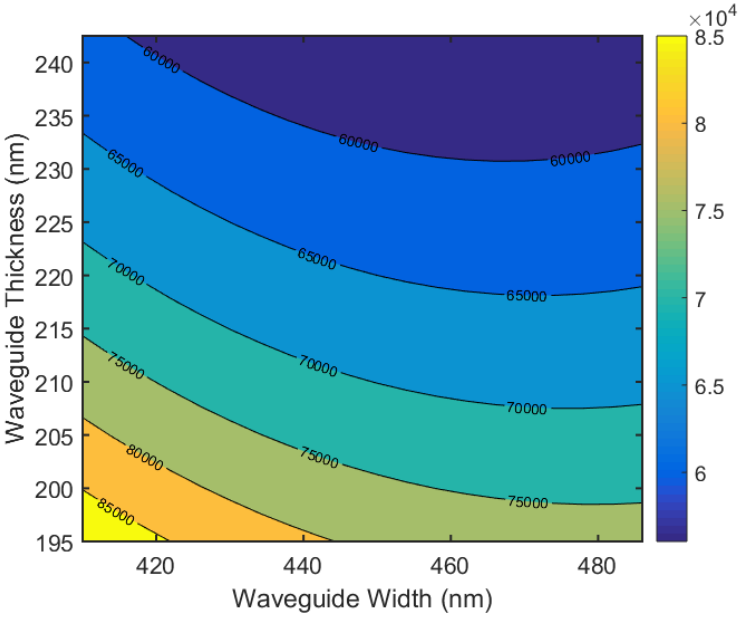

Fig. 2: 2D contour plot of field coupling coefficient vs. waveguide width and thickness.

where $\boldsymbol{\eta}=[w t]^{T}$ is the vector of the correlated random variables considered, the vector $\boldsymbol{\mu}=\left[\begin{array}{ll}w_{0} & t_{0}\end{array}\right]^{T}$ contains the corresponding nominal values (mean values) $w_{0}$ and $t_{0}$, and the matrix $\mathbf{C}$ is the covariance matrix. The symbol $\operatorname{det}(\cdot)$ represents the matrix determinant operator. The covariance matrix is defined as

$$
\mathbf{C}=\left[\begin{array}{cc}
\left(w_{0} \sigma_{w}\right)^{2} & \rho w_{0} \sigma_{w} t_{0} \sigma_{t} \\
\rho w_{0} \sigma_{w} t_{0} \sigma_{t} & \left(t_{0} \sigma_{t}\right)^{2}
\end{array}\right]
$$

where the symbols $\sigma_{w}$ and $\sigma_{t}$ are the normalized standard deviations of the $w$ and $t$, while $\rho$ is the correlation coefficient of these two random variables. The correlation coefficient $|\rho|<1$ denotes the strength of correlation: the random variables considered are independent if $\rho=0$ and strongly correlated if $|\rho|=1$. Note that, by describing this example in terms of normalized standard deviations, we make further analysis independent of the actual nominal values of our 2 random variables.

To validate the robustness of the proposed method, $\sigma_{w}$ and $\sigma_{t}$ are chosen equal to $2 \%$ and the correlation coefficient $\rho=0.9$, which is a challenging example to study since the coupling coefficient is quite dynamic with respect to the parameters considered, see Fig. 2. The proposed method is discussed in details in the following and summarized Fig. 3 .

\section{D. Variable Transformation}

Now, SC methods in the form (1) deal with independent random variables, as described in Section 2. Hence, to fit the problem into the SC framework, first of all, it is necessary to express the coupling coefficient in two independent Gaussian random variables, starting from the correlated random variables $\boldsymbol{\eta}$, defined by (9). As mentioned in Section 2, such decorrelation can be obtained via a variable transformation. Thanks to the KarhunenLoéve expansion [21], it is possible to express the vector of correlated Gaussian random variables $\boldsymbol{\eta}$ in the vector of uncorrelated Gaussian random variables with zero 


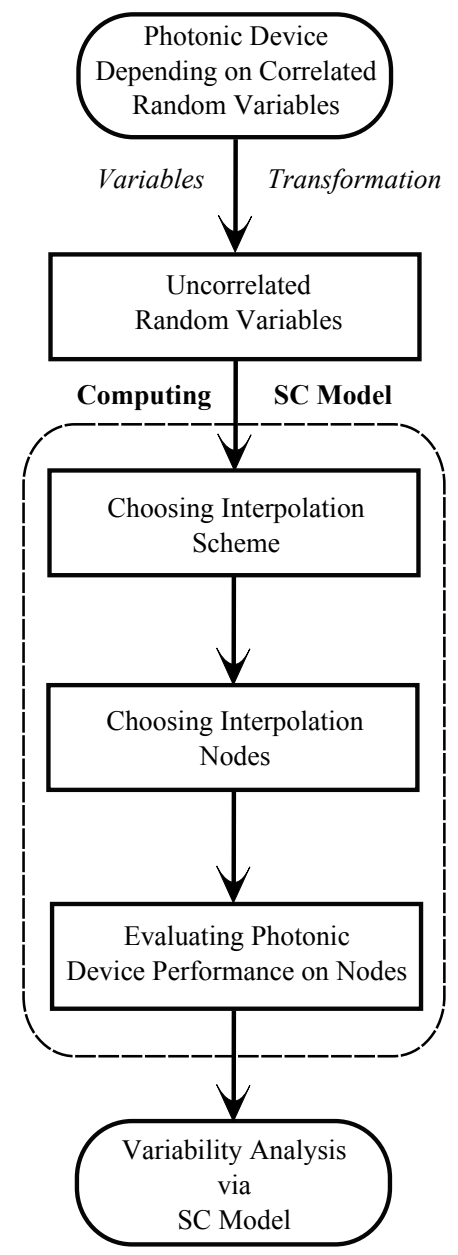

Fig. 3: Flow chart of the proposed technique.

mean and unit variance $\boldsymbol{\xi}=\left[\xi_{1}, \xi_{2}\right]^{T}$ as

$$
\boldsymbol{\eta}=\boldsymbol{\mu}+\mathrm{VE}^{\frac{1}{2}} \boldsymbol{\xi}
$$

Where $\mathbf{E}$ and $\mathbf{V}$ are the diagonal matrix of the eigenvalues and the full matrix of the eigenvectors of the covariance matrix C, respectively. Since uncorrelated Gaussian random variables are also independent, we have now expressed the coupling coefficient as a stochastic process which depends on the pair of independent Gaussian random variables $\left(\xi_{1}, \xi_{2}\right)$. An accurate description of the Karhunen-Loéve expansion for Gaussian random variables is given in Appendix 5.A.

\section{E. SC Model Computation}

To compute an SC in the form (1), the first step is choosing the interpolation scheme: the Lagrange interpolation scheme is adopted in this example for its modeling power and ease of implementation. Next, a rule which guarantees a good quality of the approximation must be used to choose the collocation points for each random variable: $\xi_{1}$ and $\xi_{2}$. In this example, the Clenshaw Curtis rule is adopted [17]: the collocation points for each random variable are the extrema of the Chebyshev polynomials.
Now, the total number of nodes could be obtained using the full tensor products of the nodes chosen for each random variable, but it would not be efficient, as discussed in Section 2. Instead, the nodes are chosen over a sparse grid based on the Smolyak algorithm. Indeed, the adoption of the Smolyak algorithm allows building our SC model by using only a subset of all the collocation points given by the full tensor product [17]. Furthermore, the collocation points chosen by the Smolyak algorithm based on the Clenshaw-Curtis rule are nested: if additional nodes are required to model the DC accurately, the nodes already computed are kept in the new sparse grid, reducing the number of evaluation of the DC coupling coefficient. See Appendix 5.B for additional details on the Smolyak algorithm. As a result, only 65 collocation points (Fig. 4) are required to build the desired SC model, and the values of the coupling coefficient at the interpolation nodes are computed using the Film Matching Mode (FMM) solver Fimmwave.

\section{F. Directional Coupler Variability Analysis}

Finally, the variability analysis for the coupling coefficient of the directional coupler under study is performed using an SC model depending on the pair of independent Gaussian random variables $\left(\xi_{1}, \xi_{2}\right)$ and the results obtained are validated through comparison with an MC analysis based on the Fimmwave FMM solver On the directional coupler cross section for the couple of correlated random variables $(w, t)$. To compare the performance of the two methods, the same set of 10000 samples for the pair of correlated random variables $(w, t)$ (see Fig. 5). The corresponding values for the independent random variables $\left(\xi_{1}, \xi_{2}\right)$ are used to estimate the device variability features.

The proposed method shows an excellent accuracy compared with the classical MC analysis, as shown in Table 1, Figs. 6, 7. In particular, the mean and the standard deviation of the coupling coefficient obtained employing the two methods are reported in Table 1: the relative error in the estimation of the mean and the standard deviation is only $9.0 \times 10^{-5}$ and $5.6 \times 10^{-3}$, respectively. Apart from stochastic moments, more complicated functions of the stochastic process under study can be estimated: the probability density and cumulative distribution function (CDF) of $\kappa$ obtained utilizing the two methods considered are in excellent agreement, as shown in Fig. 7.

\section{Table 1: Performance summary of Stochastic Collocation and Monte Carlo simulation.}

\begin{tabular}{lcc}
\hline & Monte Carlo Stochastic Collocation \\
\hline Mean value & 65160 & 65166 \\
S.t.d value & 2616.9 & 2631.4 \\
\hline
\end{tabular}

From the field coupling coefficient, we can also easily derive performance parameters of a directional coupler such as 3dB-coupling length from 

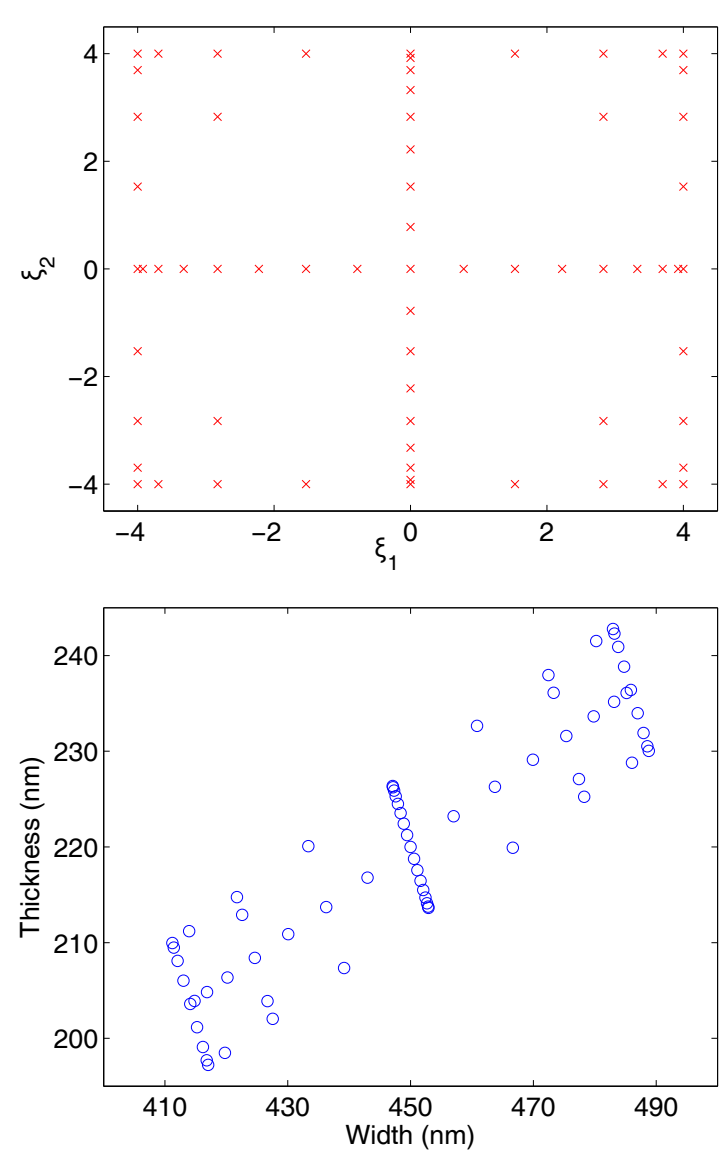

Fig. 4: Top: the red exes $(\times)$ represent the interpolation nodes for the normalized independent random variables $\xi_{1}$ and $\xi_{2}$ used to build the SC model.

Bottom: the blue circles (o) are the corresponding values for the correlated random variables $w$ and $t$ used to compute the coupling coefficients in Fimmwave.

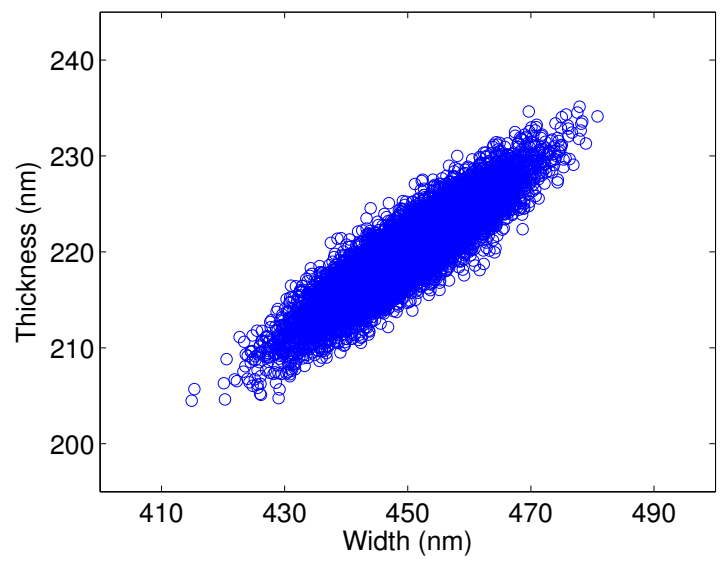

Fig. 5: Sampling points used to perform the MC analysis through direct Fimmwave simulations for the correlated random variables $(w, t)$. The corresponding values for the independent random variables $\left(\xi_{1}, \xi_{2}\right)$ are used to evaluate the SC model computed.

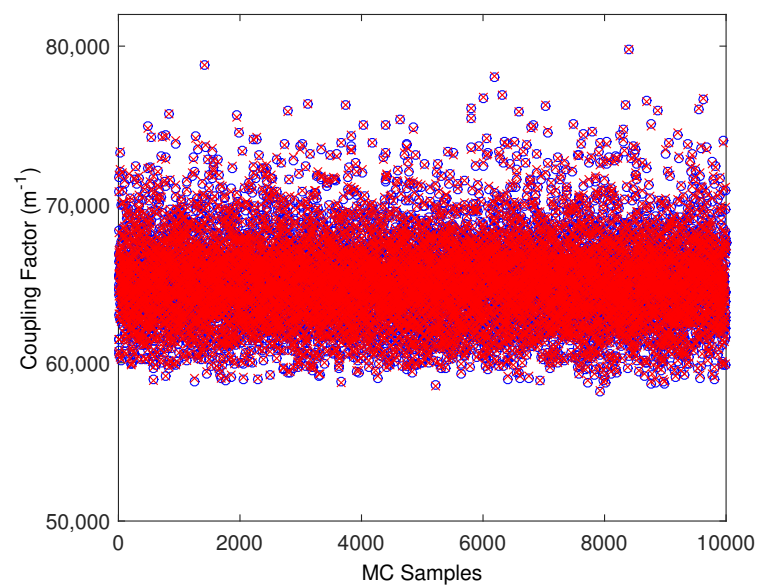

Fig. 6: Blue circles (o): coupling coefficient computed via the $\mathrm{MC}$ analysis for the $10000(w, t)$ samples shown in Fig. 5. Red $(\times)$-markers: corresponding values obtained by evaluating the SC model.

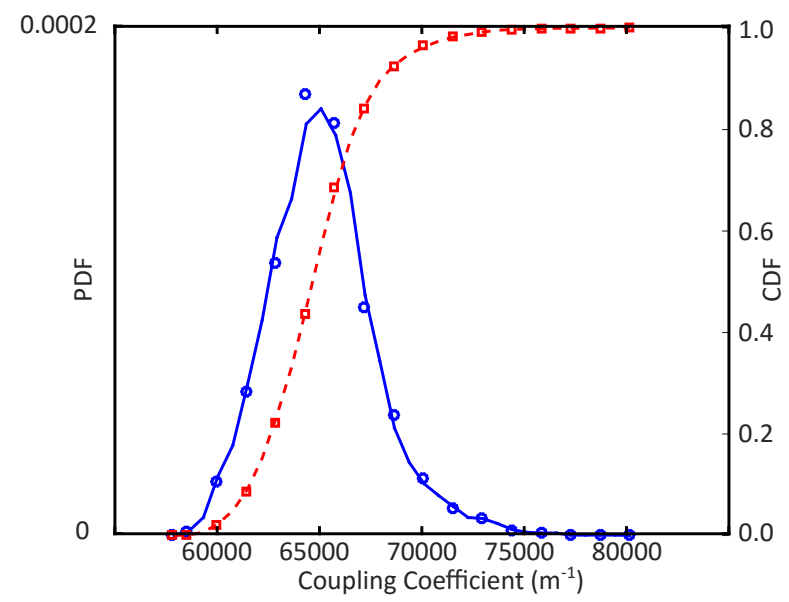

Fig. 7: The probability density function PDF and CDF of the coupling coefficient for $\lambda=1.55 \mu \mathrm{m}$. The blue solid and red dashed line are PDF and CDF obtained by means of the SC model, respectively, while the blue circles and red squares represent the same quantities computed by means of the $\mathrm{MC}$ analysis.

$$
l_{3 d B}=\arcsin (\operatorname{sqrt}(0.5)) / \kappa .
$$

As shown in Fig. 8, the proposed method shows an excellent modeling accuracy and a good estimation of the PDF and the CDF of the 3dB-coupling length $l_{3 d B}$.

Furthermore, as presented in Table 2, the SC method has dramatically saved computational cost. Note that, the SC method took a two-step procedure to perform the same variability analysis. Initially, $\mathrm{SC}$ required 65 simulations to compute the coupling coefficient at the collocation points. Next, we used the SC model over 


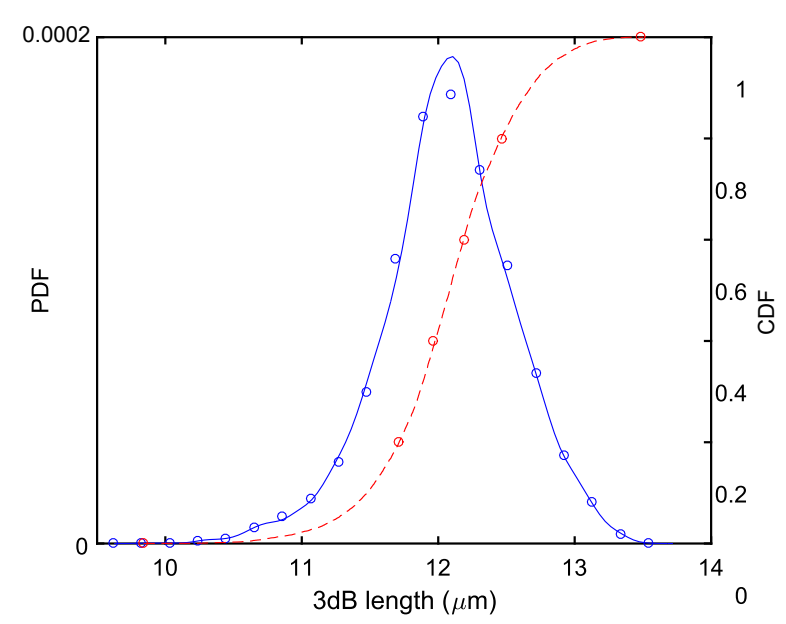

Fig. 8: The probability density function PDF and CDF of the $3 \mathrm{~dB}$-coupling length for $\lambda=1.55 \mu \mathrm{m}$. The blue solid and red dashed line are PDF and CDF obtained by means of the SC model, respectively, while the blue circles and red squares represent the same quantities computed by means of the MC analysis.

10000 samples of the independent random variables in Monte-Carlo method. Hence, the total computational time of the SC method is $8 \mathrm{~min}$ and $59 \mathrm{~s}$, which represents a speed-up of a factor $146 \times$ with respect to the MC analysis performed in Fimmwave for the couple of correlated random variables $(w, t)$, which required $21 \mathrm{~h}$ $53 \mathrm{~min} 14 \mathrm{~s}$.

\section{Conclusion}

This paper has presented the application of a novel technique for the efficient variability analysis of photonic devices, such as directional couplers. It is based on the use of stochastic collocation methods. Thanks to the flexibility in the choice of the interpolation schemes and the efficiency of sparse grid sampling to choose the collocation nodes for multiple dimension, the proposed approach is flexible and can be applied to study a broad range of photonic devices. The accuracy and efficiency of the proposed technique have been verified using comparison with the classic MC analysis for a pertinent numerical example, achieving a simulation speedup of $146 \times$.

\section{Appendix}

\section{A. Karhunen-Loéve expansion and Correlated Gaussian Random Variables}

Let us assume that the correlation matrix $\mathbf{C}^{N \times N}$ for the random variables $\boldsymbol{\eta}$ under study is symmetric and positive-definite. Then, $\mathbf{C}$ and can be diagonalized as

$$
\mathbf{C}=\mathbf{V E V}^{T}
$$

Thanks to (12), equation (9) becomes

$W_{\boldsymbol{\eta}}=\frac{1}{2 \pi \operatorname{det}(\mathbf{E})^{\frac{1}{2}}} \exp \left(-\frac{1}{2}(\boldsymbol{\eta}-\boldsymbol{\mu})^{T} \mathbf{V} \mathbf{E}^{-1} \mathbf{V}^{T}(\boldsymbol{\eta}-\boldsymbol{\mu})\right)$
Hence, the Karhunen-Loéve expansion is a simple change of variables for correlated Gaussian random variables following the non-degenerate multivariate normal distribution (9). Furthermore, it is possible to express the joint probability density function (13) with respect to a vector of independent Gaussian random variable $\boldsymbol{x}$, with zero mean and variance equal to $\left[\mathbf{E}_{i i}\right]_{i=1}^{N}$, as

$$
W_{\boldsymbol{x}}=\frac{1}{2 \pi \operatorname{det}(\mathbf{E})^{\frac{1}{2}}} \exp \left(-\frac{1}{2} \boldsymbol{x}^{T} \mathbf{E}^{-1} \boldsymbol{x}\right)
$$

where

$$
\boldsymbol{x}=\mathbf{V}^{T}(\boldsymbol{\eta}-\boldsymbol{\mu})
$$

Finally, the vector $\boldsymbol{x}$ can be written as

$$
\boldsymbol{x}=\mathbf{E}^{\frac{1}{2}} \boldsymbol{\xi}
$$

where $\boldsymbol{\xi}$ is a vector of normalized Gaussian random variables with zero mean and unitary variance. Equation (10) can be obtained by combining (15) and (16).

\section{B. Smolyak algorithm}

Let us express a stochastic process $\mathbf{Y}$ depending on one random variable $\xi$ by means of the Lagrange interpolation scheme as [16]

$$
\mathbf{U}(\xi)=\sum_{i=1}^{Q} \mathbf{Y}\left(\xi_{i}\right) L_{i}(\xi)
$$

where $L_{i}$ is given by equation (2). The $Q$ nodes can be chosen from a node distribution which guarantees a good quality of the approximation (i.e. the extrema of the Chebyshev polynomials). Extending (17) to the case of multiple random variables can be performed via tensor product, as it has been shown in Section 2, and equation (17) becomes

$$
\begin{aligned}
& \mathbf{Y}(\boldsymbol{\xi})=\mathbf{U}^{k_{1}} \otimes \cdots \otimes \mathbf{U}^{k_{N}}= \\
& \sum_{i_{1}=1}^{Q_{k_{1}}} \cdots \sum_{i_{N}=1}^{Q_{k_{N}}} \mathbf{Y}\left(\xi_{i_{1}}^{k_{1}}, \ldots, \xi_{i_{N}}^{k_{N}}\right)\left(L_{i_{1}}^{k_{1}} \otimes \cdots \otimes L_{i_{N}}^{k_{N}}\right)
\end{aligned}
$$

where $\mathbf{U}^{k_{j}}$ represents the interpolation scheme in the form (17) with respect to the random variable $\xi_{j}$ and $N$ is the number of random parameters considered. The total number of nodes required to compute (18) is the given by the product of the nodes used for each random parameter, as shown in (4). Clearly, the required number of nodes grows very quickly with respect to the number of parameters considered. Indeed, if only two nodes are used for each random variable, the total number of points required for a full-tensor product interpolation is $Q=2^{N}$.

The Smolyak algorithm allows to build multidimensional interpolation functions based on a minimal number of nodes by expressing the desired interpolation 
Table 2: Computation time of Stochastic Collocation and Monte Carlo simulation.

\begin{tabular}{|c|c|c|c|c|}
\hline \multicolumn{2}{|c|}{ Variability analysis technique } & Simulator & Number of points & Computation time \\
\hline \multicolumn{2}{|c|}{ Monte Carlo } & Fimmwave FMM Solver & 10000 & $21 \mathrm{~h} 53 \mathrm{~min} 14 \mathrm{~s}$ \\
\hline \multirow{3}{*}{$\begin{array}{c}\text { Stochastic } \\
\text { Collocation }\end{array}$} & Stochastic Modeling & Fimmwave FMM Solver & 65 & $8 \mathrm{~min} 32 \mathrm{~s}$ \\
\cline { 2 - 5 } & MC using stochastic model & SC stochastic model & 10000 & $27 \mathrm{~s}$ \\
\cline { 2 - 5 } & Total Time & & & 8 min $59 \mathrm{~s}$ \\
\hline
\end{tabular}

as a linear combination of tensor products. In particular, the property of the one-dimensional interpolation is conserved for higher dimensions. Indeed, the sparse interpolant $\boldsymbol{A}_{q, N}$ given by the Smolyak algorithm is

$$
\begin{aligned}
& \boldsymbol{A}_{q, N}(\boldsymbol{\xi})= \\
& \sum_{q-N+1 \leq|\boldsymbol{k}| \leq q}(-1)^{q-|\boldsymbol{k}|}\left(\begin{array}{c}
N-1 \\
q-\boldsymbol{k}
\end{array}\right)\left(\mathbf{U}^{k_{1}} \otimes \cdots \otimes \mathbf{U}^{k_{N}}\right)
\end{aligned}
$$

where $q-N$ is the order of interpolation, $A_{N-1, N}=0$ and $\boldsymbol{k}=\left(k_{1}, \ldots, k_{N}\right)$ with $|\boldsymbol{k}|=k_{1}+\cdots+k_{N}$. Hence, the interpolation function is built by adding a combination of one dimensional interpolant of order $k_{j}$ with the constraint that the total sum $|\boldsymbol{k}|$ across all parameters is between $q-N+1$ and $q$. Note that, $k_{j}$ can be considered as the interpolation level along the $j$-th direction.

Let us denote $\Theta$ as the set of points utilized in the onedimensional function interpolation. According to (19), the stochastic process $\mathbf{Y}$ must be computed at the nodes of the sparse grid $\mathbf{H}_{q, N}$ given by

$$
\mathbf{H}_{q, N}=\bigcup_{q-N+1 \leq|\boldsymbol{k}| \leq q} \Theta_{1}^{k_{1}} \times \cdots \times \Theta_{N}^{k_{N}}
$$

It is important to notice that, by choosing a suitable node distribution, such as Chebyshev or GaussLobatto points, the sets of collocation points $\Theta^{k}$ are nested. Hence, the sparse grid of order $q$ contains all the nodes computed for the sparse grid of order $q-1$ and the stochastic process $\mathbf{Y}$ must be evaluated only on few new collocation points.

\section{References}

[1] M. Streshinsky, R. Ding, Y. Liu, A. Novack, C. Galland, AE-J. Lim, G. Q. Lo, T. Baehr-Jones, and M. Hochberg. The road to affordable, large-scale silicon photonics. $O p$ tics and Photonics News, 24(9):32-39, 2013.

[2] W. Bogaerts, M. Fiers, and P. Dumon. Design challenges in silicon photonics. Selected Topics in Quantum Electronics, IEEE Journal of, 20(4):1-8, 2014.

[3] L. Chrostowski, X. Wang, J. Flueckiger, Y. Wu, Y. Wang, and S. T. Fard. Impact of fabrication nonuniformity on chip-scale silicon photonic integrated circuits. In Optical Fiber Communication Conference, pages Th2A-37. Optical Society of America, 2014.

[4] S. K. Selvaraja, W. Bogaerts, P. Dumon, D. Van Thourhout, and R. Baets. Subnanometer linewidth uniformity in silicon nanophotonic waveguide devices using cmos fabrication technology. Selected Topics in Quantum Electronics, IEEE Journal of, 16(1):316-324, 2010.

[5] G. S. Fishman. Monte Carlo: Concepts, Algorithms, and Applications. Springer-Verlag, New York, 1996.

[6] D. Spina, F. Ferranti, G. Antonini, T. Dhaene, and L. Knockaert. Efficient variability analysis of electromagnetic systems via polynomial chaos and model order reduction. IEEE Transactions on Components, Packaging and Manufacturing Technology, 4(6):1038 - 1051, 2014.

[7] P. R. Johnston. Defibrillation thresholds: A generalised polynomial chaos study. Conference in Computing in Cardiology, Cambridge, MA, 7-10 Sept., 2014.

[8] K. -K. K. Kim, R. D. Braatz. Generalized polynomial chaos expansion approaches to approximate stochastic receding horizon control with applications to probabilistic collision checking and avoidance. IEEE International Conference on Control Applications, Dubrovnik, Croatia, 3-5 Oct., 2012.

[9] D. Cassano, F. Morichetti, and A. Melloni. Statistical analysis of photonic integrated circuits via polynomialchaos expansion. In Advanced Photonics 2013, page JT3A.8. Optical Society of America, 2013.

[10] T. Weng, Z. Zhang, Z. Su, Y. Marzouk, A. Melloni, and L. Daniel. Uncertainty quantification of silicon photonic devices with correlated and non-gaussian random parameters. Opt. Express, 23(4):4242-4254, Feb 2015.

[11] M. S. Eldred. Recent advance in non-intrusive polynomial-chaos and stochastic collocation methods for uncertainty analysis and design. In Proc. 50th AIAA/ASME/ASCE/AHS/ASC Structures, Structural Dynamics, and Materials Conference, Palm Springs, California, May 2009.

[12] N. Agarwal and N. R. Aluru. Weighted smolyak algorithm for solution of stochastic differential equations on non-uniform probability measures. International Journal for Numerical Methods in Engineering, 85(11):13651389, 2011.

[13] N. Agarwal and N.R. Aluru. Stochastic analysis of electrostatic mems subjected to parameter variations. Journal of Microelectromechanical Systems, 18(6):1454-1468, Dec 2009.

[14] D. Xiu. Fast numerical methods for stochastic computations: a review. Communications in computational physics, 5(2-4):242-272, 2009.

[15] W. A. Weiser and S. E. Zarantonello. A note on piecewise linear and multilinear table interpolation in many dimensions. Mathematics of Computation, 50(181):253264, Jan. 1988.

[16] B. Ganapathysubramanian and N. Zabaras. Sparse grid collocation schemes for stochastic natural convection problems. Journal of Computational Physics, 225(1):652-685, 2007. 
[17] V. Barthelmann, E. Novak, and K. Ritter. High dimensional polynomial interpolation on sparse grid. $A d v$. Comput. Math., 12(4):273-288, 2000.

[18] E. Novak and K. Ritter. High dimensional integration of smooth functions over cubes. Numer. Math., 75(1):7997, 1996.

[19] E. Novak and K. Ritter. Simple cubature formulas with high polynomial exactness. Construct. Approx., 15(4):499-522, 1999.

[20] A. Der Kiureghian and P. L. Liu. Structural reliability under incomplete probability information,. J. Eng. Mech., ASCE, 112(1):85-104, 1986.
[21] M. Loeve. Probability Theory. Springer-Verlag, 4-th edition, 1977.

[22] W. Bogaerts, D. Taillaert, B. Luyssaert, P. Dumon, J. Van Campenhout, P. Bienstman, D. Van Thourhout, R. Baets, V Wiaux, and S Beckx. Basic structures for photonic integrated circuits in silicon-on-insulator. Optics Express, 12(8):1583-1591, 2004.

[23] L. Chrostowski and M. Hochberg. Silicon Photonics Design: From Devices to Systems. Cambridge University Press, 2015. 